\section{Regards sur l'économie allemande}

Bulletin économique du CIRAC

$84 \mid 2007$

Varia

\title{
Les programmes de la CDU et du SPD : convergences de fond
}

\section{Henrik Uterwedde}

\section{(2) OpenEdition}

Édition électronique

URL : http://journals.openedition.org/rea/529

DOI : $10.4000 /$ rea.529

ISBN : 978-2-8218-0864-5

ISSN : 1965-0787

\section{Éditeur}

CIRAC

Édition imprimée

Date de publication : 1 décembre 2007

Pagination : 5-12

ISSN : 1156-8992

\section{Référence électronique}

Henrik Uterwedde, «Les programmes de la CDU et du SPD : convergences de fond », Regards sur l'économie allemande [En ligne], 84 | décembre 2007, mis en ligne le 01 décembre 2009, consulté le 06 mai 2019. URL : http://journals.openedition.org/rea/529 ; DOI : 10.4000/rea.529 


\section{Les programmes de la CDU et du SPD : convergences de fond}

\section{Henrik Uterwedde}

Il y a deux ans, alors qu'ils venaient de former une grande coalition qui risquait de brouiller les repères habituels entre la gauche et la droite, SPD et CDU avaient entamé chacun un débat programmatique profond, afin de redéfinir leur profil et leur identité dans ce mariage inhabituel avec leur adversaire, mais aussi afin de renouveler leur 'boussole' face à un monde en changement rapide. Aujourd'hui, les nouveaux programmes ont été ratifiés presque en même temps, fin octobre (SPD) et début décembre (CDU) 2007. Mais le contexte politique a changé car la grande coalition allemande est entrée définitivement dans une phase plus conflictuelle, à l'approche des élections régionales en 2008 (27 janvier en Hesse et en Basse-Saxe, 24 février à Hambourg, 28 septembre en Bavière) et, déjà, à l'horizon des législatives de septembre 2009. Chacun des deux partis cherche à se démarquer de l'autre.

Or une lecture attentive des nouveaux programmes fait ressortir, une fois de plus, une large convergence, y compris dans la politique économique et sociale. C'est à partir de ce socle commun que se profilent les différences dans les approches des deux partis de gouvernement.

Drôle d'exercice que la formulation d'un programme fondamental (Grundsatzprogramm)! Après les congrès du SPD et de la CDU, les médias n'ont pas hésité à railler le caractère vague et «fourre-tout » de ces textes, qui semblent dire tout et son contraire. Pour une fois, ils ont tort, car l'objectif de ces programmes n'est justement pas de donner des indications claires et précises sur la politique envisagée mais de définir la 'carte d'identité' des partis en éclairant leurs valeurs fondamentales, en tentant de saisir les mouvements politiques, économiques et sociaux profonds et en donnant une boussole générale pour l'approche des problèmes. L'horizon de ce genre est de l'ordre de 15 à 20 ans. CDU et SPD n'en sont qu'à leur troisième exercice en la matière depuis $1945 \ldots$

\section{Cherchez le centre : les raisons d'une convergence programmatique}

"Wir sind die Mitte! ": "Le centre, c'est nous! ». Cette revendication de la Chancelière et présidente de la CDU, Angela Merkel, aux Assises de Hanovre en dit long sur l'orientation programmatique mais aussi le positionnement des partis politiques, ainsi que sur le fonctionnement de la vie politique en Allemagne. Si le SPD se définit aujourd'hui comme "parti de rassemblement de gauche ", permettant ainsi à la CDU de revendiquer la "Mitte » en exclusivité, il n'en reste pas moins que le SPD se situe au centre-gauche et les chrétiensdémocrates au centre-droit de l'échiquier politique. Car, loin de la polarisation droite-gauche et de l'idéologisation récurrente des débats en France, les principaux acteurs politiques ne redoutent pas les convergences, voire les consensus sur des questions fondamentales: l'Europe, l'économie sociale de marché, etc... Cette quête du centre s'explique par de multiples raisons.

D'abord, le caractère des partis politiques. Forts de leurs 550000 adhérents chacun, SPD et CDU sont deux grands partis de rassemblement dont

Le $3^{\mathrm{e}}$ programme fondamental pour CDU et SPD depuis 1945

SPD et CDU :

centre-gauche et centre-droit

Des partis de rassemblement 
Des débats de fond en interne

Un centrisme politique forcé par le « capitalisme rhénan »..

... le système électoral... l'audience va bien au-delà des notables, des élus et des candidats. Enracinés tous les deux dans le territoire et dans la société civile, comme dans le monde syndical et celui des entreprises, les deux partis de rassemblement sont constamment forcés d'arbitrer entre les différentes sensibilités et les multiples groupes d'intérêt qui les caractérisent. Ainsi, la CDU, réputée proche du patronat, connaît une aile syndicale et sociale, comme le SPD, issu du mouvement ouvrier, a un courant entrepreneurial et libéral. Les courants existants dans les deux partis n'ont rien à voir avec les écuries des « présidentiables » à la française ; ils sont beaucoup moins personnalisés car ils traduisent la pluralité de leurs racines idéologiques historiques : christianisme social, libéralisme et conservatisme au sein de la CDU, marxisme, socialisme démocratique, sociallibéralisme et christianisme social au SPD.

Le caractère de Volkspartei (parti de rassemblement) se traduit par un souci constant de fédérer - et, ce faisant, de dépasser - les différentes positions idéologiques et les groupes d'intérêts présents dans la société allemande. Par conséquent, ce qui distingue les deux partis - de centre-gauche et de centredroite - se manifeste bien plus souvent dans les nuances que dans les choix fondamentaux. De même, les débats de fond sur les choix de société, s'ils semblent opposer SPD et CDU dans l'espace public, sont aussi, et d'abord, menés à l'intérieur de chaque parti.

\section{Programmes fondamentaux : les précurseurs}

Ce n'est que rarement que les partis politiques se donnent le temps de réfléchir au-delà de l'horizon immédiat des prochaines élections ou d'une législature. Depuis 1945, CDU et SPD n'en sont qu'à leur troisième exercice dans ce domaine.

Le premier programme fondamental, qui reste le plus célèbre de tous, fut le «programme de Bad Godesberg » de 1959. II marqua le ralliement du SPD à l'économie sociale de marché (" autant de marché que possible, aussi peu d'Etat que nécessaire ») et aux fondements de la politique étrangère ; et, d'une manière plus générale, il fut une étape importante dans la transformation du SPD en un parti de rassemblement de gauche, inter-classes ( linke Volkspartei »), ouvrant ainsi la voie vers l'entrée au gouvernement en 1966 puis à la victoire en 1969.

Trente ans plus tard, à la veille de la chute du Mur, le programme de Berlin du SPD voté en 1989 n'a pas eu le même impact, ne réussissant pas à saisir les bouleversements économiques et sociaux de la période et ayant été dépassé rapidement par l'unité allemande. Le programme de Hambourg voté le 28 octobre 2007 est le troisième programme fondamental du SPD.

Quant à la CDU, parti de gouvernement dominant de 1949 à 1966, elle n'avait pas éprouvé le besoin de débats programmatiques approfondis. C'est après avoir perdu le pouvoir qu'elle a entrepris sa transformation en un parti de rassemblement et d'adhérents moderne, investissant massivement dans l'organisation, le recrutement d'adhérents et le travail programmatique. Le premier véritable programme fondamental, celui de Ludwigshafen (1978), fut le résultat d'un long processus de discussion sur les valeurs fondamentales mené tant à l'intérieur qu'à l'extérieur du parti, et fortement influencé par le futur président de la République, Richard von Weizsäcker. C'est à cette occasion que la CDU adopta les valeurs " liberté, solidarité, justice ».

Après l'unité, le $2^{\mathrm{e}}$ programme (Hambourg, 1994) tenta de tenir compte des mutations économiques et sociales en cours en cherchant à doter l'économie sociale de marché d'une dimension écologique, et en abordant la transformation des systèmes de sécurité sociale. II est maintenant remplacé par le programme adopté à Hanovre le 3 décembre 2007.

Ensuite, la culture politique allemande 'centriste': Elle abhorre les extrémismes de gauche comme de droite (rappelons qu'au niveau national, l'extrême-droite et l'extrême-gauche restent complètement marginalisées par les électeurs) et préfère le pragmatisme et le compromis aux "ruptures ». Ceci explique également les larges convergences entre CDU et SPD. Pour sa part, le capitalisme rhénan, par sa volonté de concilier les impératifs de la compétitivité avec la cohésion sociale, et en mettant en avant la coopération et la négociation sociale comme agents de la régulation à côté de la dynamique du marché, est à la fois le résultat et le vecteur de ce large consensus sur l'économie sociale de marché.

S'y ajoute le fait que le centrisme des deux grands partis est aussi porté par des facteurs institutionnels. Dans un système politique dominé par le vote à la proportionnelle qui condamne les grands partis à chercher des partenaires afin 
d'obtenir une majorité de gouvernement, CDU et SPD sont amenés à occuper une place au centre, afin d'élargir les choix possibles de partenaires. Ainsi, le SPD, qui a gouverné avec les Verts de 1998 à 2005, cherche à ne pas couper les ponts avec le parti libéral FDP (son partenaire de coalition de 1969 à 1982) dont il pourrait avoir besoin demain. Quant à la CDU, si elle préfère une alliance avec le FDP (comme dans les gouvernements de Helmut Kohl entre 1982 et 2002), elle commence, non sans mal, à tester l'éventualité d'une possible coalition avec les Verts. II va sans dire qu'une grande coalition CDU-SPD (comme en 1966-1969 et depuis 2005), même si elle est évitée pour des raisons de fonctionnement démocratique, reste une option tout à fait réaliste si elle s'impose par les résultats électoraux comme en 2005. Pour autant, il ne faut pas en conclure que ces calculs de pouvoir des acteurs politiques l'emportent systématiquement sur les considérations programmatiques, et que ces dernières n'auraient qu'un intérêt secondaire. En général, les coalitions et alliances politiques sont fondées sur des convergences programmatiques permettant de s'entendre sur un programme de législature. Ce qui est facilité par le caractère centriste et ouvert des programmes.

Enfin, l'enseignement de la politique des réformes structurelles des dernières années a rappelé aux deux grands partis la nécessité d'équilibrer leurs politiques et leurs discours. Le SPD du chancelier Schröder a payé un prix politique lourd pour les réformes courageuses de l'Agenda 2010: une partie de sa base syndicale, de ses adhérents et de son électorat l'ont déserté, il a perdu (quoique de peu) les législatives de 2005 et doit désormais faire face à un parti sur sa gauche: Die Linke, adepte d'un «toujours plus » populiste. D'où une tentative de reconquête qui cherche à trouver des accents plus sociaux dans le positionnement de centre-gauche qui reste celui du SPD, et qui s'exprime dans sa définition comme "parti de rassemblement de gauche » (linke Volkspartel). Quant à la CDU, elle avait mené une campagne électorale jugée trop " froide » et "néolibérale » en 2005 , qui était basée sur le très libéral programme de Leipzig de 2003 et sur l'entente avec le FDP néolibéral. Mais elle a failli perdre une élection qu'elle croyait déjà largement gagnée d'avance parce que l'électorat restait méfiant vis-à-vis d'un discours de la rupture libérale.

Depuis, la CDU a cherché à se recentrer et à mieux équilibrer ses approches, comme le témoigne le discours d'Angela Merkel aux Assises de Hanovre: " une politique du centre dit à tous les membres de la société : pensez toujours aux autres aussi ! N'oubliez pas que nous dépendons les uns des autres! Des entreprises prospères n'existent que sur des sites de production performants, et des sites performants n'existent que s'il y a justice sociale et sens de la responsabilité... L'économie sociale de marché, c'est toujours une économie et une société qui incluent tout le monde. Si cela ne fonctionne plus, toute la boutique sautera en l'air, pour le dire d'une manière très simple ". D'où cette politique du juste milieu qui, comme l'a fait Angela Merkel, renvoie dos à dos le libéralisme économique ("qui veut tout laisser au libre jeu du marché ") et le protectionnisme ("qui, par peur de la globalisation, veut ériger toujours de nouvelles barrières") comme solutions du passé, pour lui opposer une "approche holistique " qui inclut "liberté, concurrence et esprit entrepreneurial d'un côté, participation, responsabilité et solidarité de l'autre ». Des phrases qui pourraient être souscrites par la grande majorité des Allemands. On peut en conclure que le centrisme des grands partis correspond profondément aux attentes de la société allemande.

\section{Un socle commun : les fondements du modèle économique et social}

Par conséquent, il n'est pas surprenant que la lecture des deux programmes fondamentaux dégage un certain nombre de convergences profondes dans les domaines de la politique économique et sociale.
... et l'enseignement de l'action politique 
Des valeurs partagées

Une doctrine partagée : l'économie sociale de marché

Une vision consensuelle de l'Europe
Cela concerne d'abord les valeurs. Si la CDU se déclare toujours guidée par une vision chrétienne de l'Homme tandis que le SPD se réfère de nouveau à sa tradition du socialisme démocratique, les deux partis partagent de manière explicite les valeurs fondamentales : liberté, justice, solidarité - des valeurs qui forment une unité et se conditionnent, se complètent, se renforcent et se limitent mutuellement, comme l'expriment les deux programmes de manière presque identique. Ce qui semble abstrait est en vérité une convergence importante, qui exclut toute stratégie de type «thatcherienne » au profit d'un réformisme gradualiste.

Cette convergence se vérifie quand on compare l'approche des deux partis concernant le modèle économique et social, autrement dit l'économie sociale de marché. Pour la CDU, qui en réclame le droit d'auteur à juste titre, ce modèle est caractérisé par "l'unité indivisible d'un ordre économique libéral et d'un ordre social solidaire ", qui tire sa force du fait que "liberté et responsabilité, concurrence et solidarité forment un ensemble ", tandis que le SPD y voit " un modèle de réussite exemplaire » parce qu'il "combine la force économique avec le bien-être de larges couches de la société ». Si le poids respectif du social et du libéral dans le modèle fait objet de différentiations (pas seulement entre CDU et SPD mais aussi à l'intérieur de chacun d'entre eux), il est significatif à quel degré les deux grands partis soulignent les mêmes éléments constitutifs de l'économie sociale de marché : culture de stabilité et importance de la consolidation budgétaire, liberté des entreprises combinée à une responsabilité sociale, nécessité d'une culture entrepreneuriale et importance du Mittelstand (PME), démocratie sociale via l'autonomie dans le domaine des conventions collectives, cogestion et participation des travailleurs, primauté des marchés mais légitimité d'intervention des pouvoirs politiques dans des domaines et sous des formes bien définis, sécurité sociale pour tous guidée par les principes de la solidarité collective et la responsabilité individuelle...

Quant à la politique de compétitivité, elle aussi est largement partagée par les deux partis. Les fondements du modèle de compétitivité et du capitalisme coopératif à l'allemande - salaires maîtrisés mais élevés, capacité d'innovation, pôles décentralisés de compétitivité, tissu d'entreprises marqué par la force du Mittelstand (surtout les grosses PME), implication et participation des salariés font partie d'un large consensus, tout comme les priorités d'action dans ce domaine : développement d'une culture entrepreneuriale, nouvelle priorité pour les investissements tant financiers qu'organisationnels dans le savoir : l'éducation, du préscolaire au niveau universitaire, la formation, la recherche, les mécanismes de transfert de technologies, les financements des créateurs d'entreprises innovantes... CDU et SPD favorisent une approche d'action publique multi-acteurs, transversale, partenariale, et bottom-up, donnant toute leur place aux acteurs de l'économie.

Consensus très marqué aussi dans la vision de l'Europe : une Europe politique forte, capable d'être une réponse à la globalisation ; une Europe porteuse d'un modèle économique et social proche de l'économie sociale de marché ; une Europe citoyenne dans une vision très fédérale, mettant l'accent sur le rôle des institutions, notamment un Parlement européen renforcé, mais aussi sur les acteurs (partis politiques au niveau européen, opinion publique européenne), ainsi que sur le principe de subsidiarité. Ce n'est que sur l'Europe économique que les discours se différentient, la CDU mettant l'accent sur l'union monétaire et le Marché unique et mettant en garde contre toute sur-réglementation, tandis que le SPD prône une « Europe sociale » caractérisée par " des règles et standards européens contraignants » et un certain nombre d'éléments comme les droits des salariés, la cogestion, l'autonomie du champs des conventions collectives, des taux minima dans l'imposition des entreprises, etc. Toujours est-il que là aussi, les visions restent assez proches, que l'appui fort de la CDU pour l'indépendance de la BCE et le Pacte de stabilité et de croissance est partagé par le $\mathrm{SPD}$, et que l'appel à la consolidation des finances publiques figure dans les 
deux programmes. Notons pourtant le différend clair sur l'adhésion de la Turquie, refusée par la CDU au nom d'une solution alternative de «partenariat privilégié » tandis que le SPD veut donner à la Turquie la chance de prouver sa capacité à remplir les critères d'adhésion de l'Union.

La vision de la globalisation fait aussi partie du socle commun entre CDU et SPD. Si les deux partis peuvent mettre des accents différents, ils sont largement d'accord pour considérer la globalisation comme un phénomène comportant des chances et des risques, et comme un défi au capitalisme rhénan qui peut et doit être relevé à deux niveaux : au niveau national, par une adaptation de l'économie allemande pour qu'elle puisse pleinement profiter des chances de la globalisation ; au niveau européen et international, par la mise en place d'un cadre réglementaire international. Les maîtres mots : " gestion de la globalisation » et " cadre réglementaire mondial », renvoient par ailleurs à la tradition ordo-libérale allemande développée après 1945, qui stipule qu'il n'y a pas de marché performant sans encadrement réglementaire.
Une appropriation commune de la globalisation

\section{Une vision largement convergente de la mondialisation}

CDU, Programme de Hanovre, décembre 2007 :

«La globalisation est une évolution générée par l'Homme, que nous pouvons influencer et façonner. Si nous veillons à ce que notre politique soit économiquement raisonnable et socialement juste, la globalisation ne scellera pas la fin de l'Etat social ni de l'économie sociale de marché. Notre politique pourra au contraire déclencher son acceptation mondiale. Une nouvelle politique économique doit ancrer les standards de l'économie sociale de marché à l'échelle internationale. C'est ainsi que l'économie sociale de marché atteindra une nouvelle dimension ".

"Ce n'est pas par le cloisonnement et la sur-règlementation que nous allons sauvegarder la force de l'économie sociale de marché, mais par davantage de liberté et de concurrence ».

"L'Union européenne, communauté économique et communauté de valeurs, est un modèle prouvant que l'ouverture des marchés et la concurrence peuvent fonctionner tout en sauvegardant les identités nationales et en prenant en compte les standards juridiques, sociaux et écologiques au service de tous. En même temps, l'Europe ... est un acteur puissant pour la mise en œuvre d'un cadre réglementaire juste pour l'économie mondiale. Nous voulons renforcer la capacité de l'UE à accomplir cette tâche importante pour l'avenir... Nous oeuvrons pour le renforcement de la capacité d'action, de la transparence et de l'efficacité des organisations internationales existantes. 》

SPD, Programme de Hambourg, octobre 2007 :

«La globalisation, l'ouverture des frontières et des marchés, n'est pas seulement le résultat d'innovations technologiques, mais aussi de décisions politiques... Mais en même temps, le capitalisme global se caractérise par un manque de démocratie et de justice. Ainsi, il s'oppose à l'objectif d'un monde libre et solidaire. ”

«Nous considérons la globalisation pour surmonter la faim, la pauvreté et autres fléaux. Car le commerce mondial génère de nouveaux emplois et la prospérité pour le plus grand nombre. Nous voulons mettre cette dynamique des marchés au service de l'Homme. Pour ce faire, nous avons besoin d'un cadre réglementaire pour la concurrence, qui déploie la croissance à long terme et dépasse la fixation sur le profit à court terme. "

«Avec la globalisation, le monde devient un marché d'un seul tenant. Le pouvoir économique se concentre entre les mains d'entreprises, de banques et de fonds agissant au niveau mondial. Les multinationales cherchant à réaliser leurs profits par-delà les frontières, leurs stratégies font fi des décisions démocratiquement fondées. Les Etats, même les plus grands, risquent de devenir de simples sites rivalisant pour attirer les investissements de ce capital globalisé. C'est pourquoi les Etats doivent s'unir pour renforcer leur influence. L'Europe a emprunté cette voie. Une Europe sociale peut devenir le modèle pour d'autres parties du monde. »

\section{Un modèle, deux approches}

Cela dit, ces convergences profondes ne constituent pas un consensus mou. Car à partir de ce socle commun, les approches des deux partis se distinguent suffisamment pour proposer deux voies alternatives. Ainsi, la CDU met davantage l'accent sur le rôle des entreprises et des marchés que le SPD. Dans la tradition ordo-libérale, elle définit la fonction de l'Etat comme destinée à faire fonctionner les marchés, en développant le cadre réglementaire nécessaire, et reste plus réticente quant à d'autres types d'interventionnisme économique. Dans ce contexte, elle rend un hommage appuyé au rôle crucial des entreprises, qui doivent être encouragées par un cadre plutôt libéral et non contraignant, et elle s'en prend à la sur-réglementation en matière économique. Le SPD, lui, reste tributaire d'une vision d'un Etat plus actif, tant dans le domaine réglementaire que dans la politique macroéconomique ou industrielle (mais qui, en fait, est définie comme une politique du site de production), sans pour autant céder à un étatisme à la française.

Deux approches de l'articulation Etat/marché 


\begin{tabular}{|c|c|c|}
\hline \multicolumn{3}{|c|}{ Deux approches de la politique économique et sociale } \\
\hline & SPD & CDU \\
\hline $\begin{array}{l}\text { Rôles de l'Etat } \\
\text { et du marché }\end{array}$ & $\begin{array}{l}\text { "... nous insistons sur le primat de la politique dé- } \\
\text { mocratique et récusons la soumission du politique à } \\
\text { l'économique. Ce faisant, nous avons une notion } \\
\text { large du politique qui ne se réduit pas à l'Etat mais } \\
\text { inclut les alliances et réseaux de la société civile } \\
\text { ainsi que l'action libre et autonome de chacun. " } \\
\text { "L'Etat ne peut ni ne doit se substituer aux marchés. } \\
\text { Mais il peut donner des impulsions aux marchés lea- } \\
\text { ders. Il doit donner des priorités en matière de } \\
\text { politique industrielle et se concentrer sur des champs } \\
\text { stratégiques, en partenariat avec les acteurs écono- } \\
\text { miques et le monde scientifique. " } \\
\text { "Une politique industrielle stratégique mise sur le } \\
\text { développement des avantages qualitatifs de notre } \\
\text { site de production. Elle renforce les pôles industriels } \\
\text { et les compétences économiques régionales. " } \\
\text { "Notre politique financière et monétaire, en Alle- } \\
\text { magne et en Europe, vise à stabiliser la conjoncture } \\
\text { et à favoriser une croissance régulière et forte. " } \\
\text { "Pour nous, le marché est non seulement un moyen } \\
\text { nécessaire, mais aussi meilleur que d'autres méca- } \\
\text { nismes de coordination. Mais abandonné à lui- } \\
\text { même, le marché est aveugle au plan social et } \\
\text { écologique... Pour qu'il puisse déployer son effica- } \\
\text { cité positive, le marché a besoin de règles, d'un Etat } \\
\text { capable d'agir et de sanctionner, de lois efficientes et } \\
\text { de prix fixés selon les règles du fair play. " }\end{array}$ & $\begin{array}{l}\text { "L'économie sociale de marché est plus qu'un sys- } \\
\text { tème économique. C'est un modèle de société. Elle } \\
\text { crée des conditions cadres dans lesquelles les } \\
\text { forces créatrices des individus peuvent se déployer à } \\
\text { l'unisson. " } \\
\text { "Dans l'économie sociale de marché, la politique est } \\
\text { une politique qui crée un cadre réglementaire } \\
\text { [Ordnungspolitik]... Elle permet à chacun une pré- } \\
\text { sence active sur le marché. Elle présuppose que } \\
\text { l'Etat garantisse les conditions du libre exercice de la } \\
\text { concurrence. En font partie la liberté d'entreprendre } \\
\text { et de contracter, la protection contre des barrières } \\
\text { d'accès au marché et contre l'abus de position } \\
\text { dominante d'entreprises en situation de monopole, } \\
\text { ainsi que la transparence sur les marchés " } \\
\text { "La CDU défend depuis toujours une politique fon- } \\
\text { dée sur la confiance dans la force créatrice de mar- } \\
\text { chés libres et d'une concurrence loyale... Le principe } \\
\text { directeur doit être le principe de la subsidiarité : aussi } \\
\text { peu d'Etat que possible, autant d'Etat que néces- } \\
\text { saire. " } \\
\text { "La compétitivité d'une économie vit du dynamisme } \\
\text { de ses entreprises... La mission de l'Etat est donc de } \\
\text { soutenir les créateurs et les petites et moyennes } \\
\text { entreprises grâce à des conditions-cadre optimales. } \\
\text { Davantage de marché signifie davantage de chances } \\
\text { pour de nouvelles créations d'entreprises. Nous vou- } \\
\text { lons diminuer la part de l'Etat dans le PIB, réduire la } \\
\text { bureaucratie, rendre le droit du travail transparent et } \\
\text { flexible et le système fiscal compétitif, et améliorer } \\
\text { les conditions pour le financement des entreprises. " }\end{array}$ \\
\hline Travail/emploi & $\begin{array}{l}\text { «La politique... du plein emploi repose sur quatre } \\
\text { piliers : une croissance forte et qualitative [mettant } \\
\text { l'accent sur l'innovation et le dynamisme du secteur } \\
\text { tertiaire]; ... une politique transversale [emploi, } \\
\text { famille, formation et parité] qui aide chacun à maîtri- } \\
\text { ser les ruptures et transitions dans sa vie profession- } \\
\text { nelle; ... des emplois aidés et d'utilité publique pour } \\
\text { ceux qui n'ont pas de perspective sur le marché du } \\
\text { travail; une politique moderne du temps de travail } \\
\text { favorisant flexibilité et autonomie des salariés et } \\
\text { permettant l'accès à l'emploi à un plus grand nombre } \\
\text { par une réduction du temps de travail. " } \\
\text { "Nous luttons pour des salaires minima garantissant } \\
\text { la subsistance en Allemagne et en Europe, et qui } \\
\text { doivent être déterminés par la négociation sociale et } \\
\text { la loi. " }\end{array}$ & $\begin{array}{l}\text { "Le chômage en Allemagne a principalement des } \\
\text { origines structurelles. C'est pourquoi nous n'avons } \\
\text { pas seulement besoin d'une croissance durable et } \\
\text { plus élevée, mais aussi d'investissements ciblés } \\
\text { dans l'éducation et d'une réforme de fond du marché } \\
\text { du travail... Sur le marché du travail, il convient de } \\
\text { trouver un compromis équitable entre le besoin de } \\
\text { protection des salariés, les chances des chômeurs } \\
\text { de trouver un emploi et les intérêts des entreprises... } \\
\text { Une flexibilisation de la protection contre le licencie- } \\
\text { ment, qui améliore les chances des chômeurs, est } \\
\text { de ce fait un impératif de justice sociale. " }\end{array}$ \\
\hline $\begin{array}{l}\text { Justice sociale, } \\
\text { sécurité sociale }\end{array}$ & $\begin{array}{l}\text { "L'Etat social de prévoyance [vorsorgender Sozial- } \\
\text { staat]": "celui-ci combat la pauvreté et rend } \\
\text { chacun capable de gérer sa vie de manière } \\
\text { autonome. II soutient le travail qui garantit la } \\
\text { subsistance, aide dans l'éducation et mise sur la } \\
\text { prévention médicale. " }\end{array}$ & $\begin{array}{l}\text { "La société des chances [Chancengesellschaft)] ": } \\
\text { " nous refusons une vision de la sécurité sociale et } \\
\text { de la solidarité qui serait uniquement fondée sur des } \\
\text { transferts financiers. Seule la combinaison entre res- } \\
\text { ponsabilité individuelle, prestations publiques et en- } \\
\text { gagement civique est à même de créer la sécurité } \\
\text { dont les Hommes ont besoin." }\end{array}$ \\
\hline Fiscalité & $\begin{array}{l}\text { "La démocratie sociale requiert un Etat capable } \\
\text { d'agir. Seuls les riches peuvent se permettre un Etat } \\
\text { pauvre. Or pour donner corps à sa politique, l'Etat } \\
\text { doit disposer de ressources suffisantes et pé- } \\
\text { rennes. " } \\
\text { "Les revenus et les fortunes ne sont pas répartis de } \\
\text { manière équitable. La politique fiscale social- } \\
\text { démocrate doit limiter les inégalités et favoriser } \\
\text { l'équité des chances... Nous sommes attachés à } \\
\text { l'impôt progressif sur les revenus. Nous voulons une } \\
\text { imposition juste des grandes fortunes et héritages. " }\end{array}$ & $\begin{array}{l}\text { "Pour que les citoyens acceptent le droit fiscal, il } \\
\text { doit suivre les principes suivants : se caractériser par } \\
\text { des taux faibles, être simple et juste..., ceux qui sont } \\
\text { financièrement plus performants doivent contribuer } \\
\text { plus que les autres. En même temps, le droit fiscal } \\
\text { ne doit pas restreindre par des taux exagérés la li- } \\
\text { berté des choix budgétaires des citoyens. Un régime } \\
\text { fiscal avec des faibles taux d'imposition encourage } \\
\text { l'effort et l'initiative tout en permettant à chacun de } \\
\text { se prémunir contre les risques de la vie. " }\end{array}$ \\
\hline
\end{tabular}

Source : Compilation de l'auteur à partir des programmes fondamentaux des deux partis. 
La fiscalité constitue un autre domaine de différentiation. La CDU renoue (un peu timidement) avec sa campagne de 2005 sur le thème « trop d'impôt tue l'impôt » en demandant une réforme du système fiscal pour le rendre plus simple, plus modeste (avec des taux d'imposition encore en baisse) et plus juste. Le SPD, lui, souligne les fonctions multiples des pouvoirs publics et la nécessité d'un financement suffisant. II assigne au système fiscal l'objectif de limiter les inégalités des revenus et des fortunes, ce qui implique des choix fiscaux différents de ceux de la CDU. C'est ici que se fait sentir que la base sociale des deux partis, si elle est composite dans les deux cas, reste différentiée : celle de la CDU repose davantage sur les milieux aisés et le monde de l'entreprise, celle du SPD sur les classes moyennes et le salariat.

La sociologie des deux partis peut expliquer aussi un autre clivage, concernant la politique salariale, notamment pour les moins qualifiés. La CDU penche pour des salaires correspondant à la faible productivité de ces salariés, qui seraient moins pénalisants pour l'emploi, et pour des primes additionnelles payées par les pouvoirs publics. Le SPD souligne que chaque emploi à plein temps doit couvrir les besoins de subsistance des salariés, et revendique l'instauration de salaires minima par la négociation sociale ou par voie législative. Le laborieux compromis qu'a trouvé la grande coalition sur l'introduction d'un salaire minimum dans les services postaux témoigne de ce clivage : il est rejeté vivement par les milieux d'affaires et la base patronale de la CDU parce qu'il cimenterait le monopole de la Deutsche Post et détruirait des emplois, et le reproche à la chancelière d'avoir trop concédé au SPD n'est pas loin. Ceci amène la CDU à en faire un cas d'exception alors que le SPD vise à élargir ce mécanisme à d'autres secteurs économiques et semble décidé d'en faire un thème central de la future campagne électorale.

La politique de l'emploi oppose elle aussi le SPD, qui tient à la protection existante contre les licenciements, à la CDU, qui veut y introduire une souplesse nouvelle, censée être favorable à la création de nouveaux emplois.

En ce qui concerne la sécurité sociale, malgré une certaine convergence entre le concept de la Chancengesellschaft (société des chances, qui donne la priorité à l'équité des chances sur l'égalité des résultats) de la CDU et le Vorsorgender Sozialstaat (Etat social de prévoyance, qui élargit l'Etat social vers une action préventive, transversale en activant les individus), et malgré le souci commun de découpler davantage le financement de la sécurité sociale des revenus du travail afin de baisser les coûts du travail, les clivages restent quant au financement. Ainsi, dans l'assurance-maladie, le concept de la CDU, qui prône une prime unique pour chaque citoyen (Gesundheitsprämie), est incompatible avec l'approche du SPD, qui se prononce pour une CSG à la française (Bürgerversicherung). C'est cette incompatibilité qui avait débouché sur la création d'un Fonds Santé (Gesundheitsfonds ; voir REA 79/06) dont l'entrée en vigueur est théoriquement prévue pour 2009. De manière plus générale, la vision de la CDU reste plus libérale quand elle déclare que « dans tous les systèmes de protection sociale, il faut renforcer la concurrence, la transparence, l'efficience, la conscience des coûts de la protection, le sens de la responsabilité et le principe de subsidiarité. »

SOMME TOUTE, LA COMPETITION ENTRE CDU ET SPD, entre centre-droit et centregauche, est bien vivante. Elle repose sur deux visions de l'économie, de la société et de l'action publique qui se distinguent sans être séparées par un fossé. L'existence d'un socle commun partagé par les deux partis - comme par la plupart des autres, la gauche socialiste exceptée - et l'absence de polarisations idéologiques lourdes permettent un véritable débat sur la meilleure politique, évitant des manichéismes et des guerres idéologiques stériles. Ceci n'exclut nullement des mouvements de rejet de réformes ni des réflexes protectionnistes dans la société comme on l'a constaté à plusieurs reprises ces dernières années, par exemple dans le rejet de la libre circulation des travailleurs venant
Des divergences sur la fiscalité...

... les salaires et l'emploi

Opposition sur la protection sociale 
des nouveaux Etats membres de l'UE (les «carreleurs polonais " jouant le même rôle d'épouvantail outre-Rhin que les « plombiers polonais " en France). Réaliser des changements qui heurtent les «acquis " des uns et des autres reste une tâche difficile, en Allemagne comme ailleurs. II n'empêche que le pragmatisme et la convergence des approches qui se dégagent à la lecture des programmes fondamentaux de la CDU et du SPD facilitent des constats partagés, des négociations collectives et des processus de décisions permettant d'engager les changements nécessaires.

\section{Indications bibliographiques}

CDU : Grundsätze für Deutschland, www.hannover2007.cdu.de/download/071203-beschlussgrundsatzprogramm-4.pdf

Discours d'ouverture d'Angela Merkel, www.hannover2007.cdu.de/download/071203-redemerkel-final.pdf

SPD : Hamburger Programm. Das Grundsatzprogramm der SPD. Beschlossen am 28. Oktober 2007 auf dem SPD-Parteitag in Hamburg, www.parteitag.spd.de/servlet/PB/show/ 1731523/Hamburger\%20Programm_final.pdf

Commun P. (dir.), L'ordolibéralisme allemand. Aux sources de l'économie sociale de marché, Travaux et Documents du CIRAC, Cergy-Pontoise, 2003

VON HAUfF, M. (dir.), Die Zukunftsfähigkeit der Sozialen Marktwirtschaft, Marburg, 2007

LAUK K. (dir.), Was würde Ludwig Erhard heute sagen ? Stuttgart/Leipzig, 2007

STEINBRücK P., « Die Zukunft der sozialen Demokratie - die Rolle des Staates », discours à la Fondation Friedrich Ebert, Potsdam, 7 juin 2006 (www.programmdebatte.spd.de)

UTERWEDDE H., " CDU et SPD à la recherche de nouvelles orientations », Regards sur l'économie allemande, 78/2006

UTERWEDDE H., "'Capitalisme rhénan' : défis d'adaptation et compétitivité virtuelle » in BOURGEOIS I. (dir.), Le modèle social allemand en mutation, Travaux et Documents du CIRAC, Cergy-Pontoise, 2005

UTERWEDDE H., "Les Allemands face à la mondialisation », in FABRY E. (dir.), Les Européens face à la mondialisation, Paris, 2007, www.fondapol.org/v2/pdf/Etude_Mondialisation. pdf. 